Supporting Information accompanying:

\title{
$\pi$-hole interactions involving nitro compounds: directionality of nitrate esters
}

Antonio Báuza, ${ }^{a}$ Antonio Frontera*, and Tiddo J. Mooibroek ${ }^{*, b}$

${ }^{a}$ Department of Chemistry, Universitat de les Illes Balears, Crta. de Valldemossa km 7.5, 07122 Palma (Baleares), SPAIN; Fax: (+) 34971 173426; E-mail: toni.frontera@uib.es

bvan't Hoff Institute for Molecular Sciences, Universiteit van Amsterdam, Science Park 904, 1098 XH Amsterdam, The Netherlands; E-mail: t.j.mooibroek@uva.nl 


\section{Table of Contents:}

1. Additional computational details ......................................3

1.1. AIM analysis of selected complexes....................................................

1.2. Data on structural isomers of some $\mathrm{R}-\mathrm{NO}_{2}$ molecules.........................3

1.3. Cartesian coordinates of optimized geometries ...................................4

2. CSD Analysis..............................................................14

2.1. Description of queries used to retrieve data from the CSD ................14

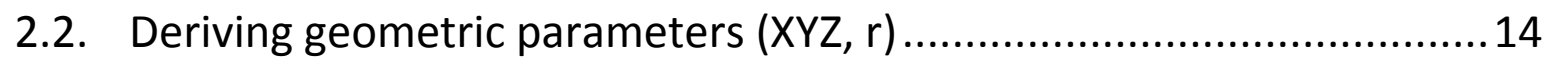

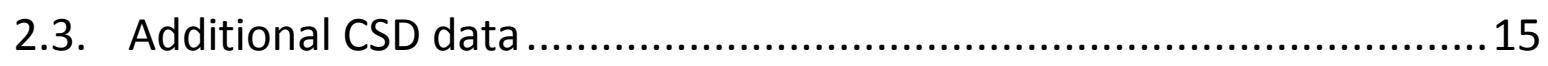




\section{Additional computational details}

\subsection{AIM analysis of selected complexes}

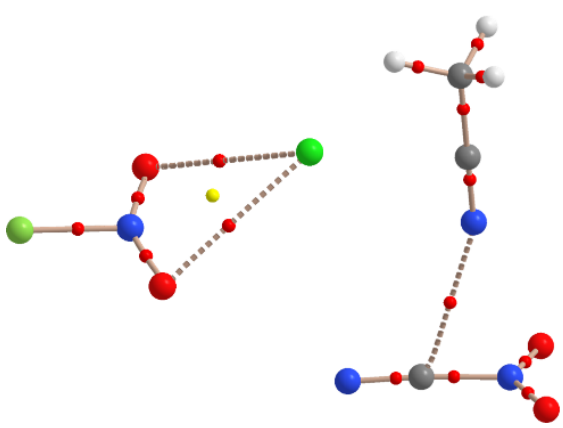

$2+\mathrm{Cl}^{-}$

$6+\mathrm{NCCH}_{3}$

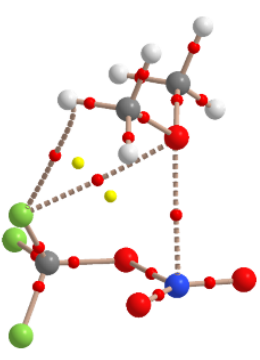

$8+\mathrm{O}\left(\mathrm{CH}_{3}\right)_{2}$

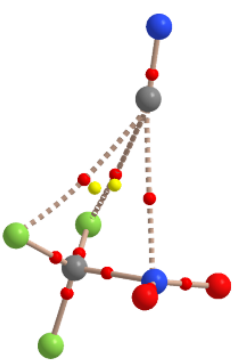

$10+\mathrm{NC}^{-}$

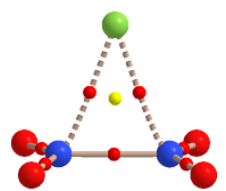

$12+\mathrm{Cl}^{-}$

Figure S1. Graphical renderings of an 'Atoms in Molecules' analysis for several selected complexes, computed at the MP2/6-311+G** level of theory using the AIMAll program. ${ }^{1}$

\subsection{Data on structural isomers of some $\mathrm{R}-\mathrm{NO}_{2}$ molecules}

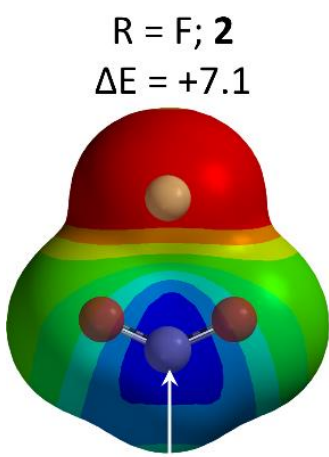

$+35$

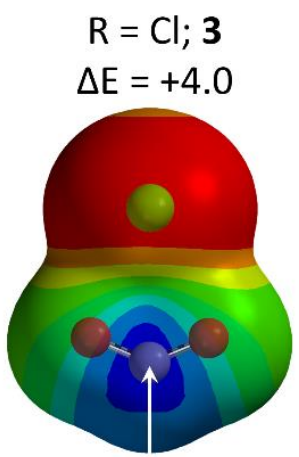

$+43$

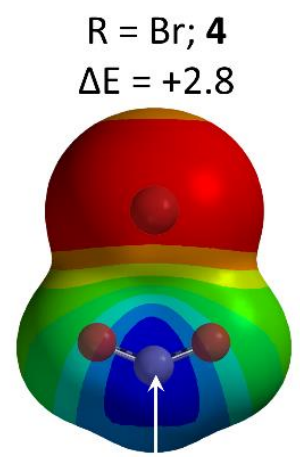

$+47$

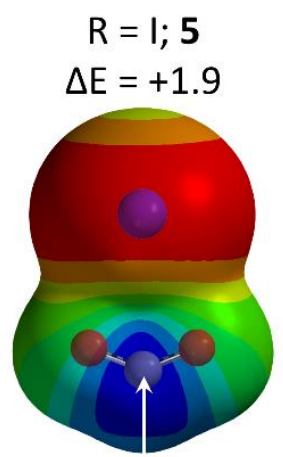

$+47$

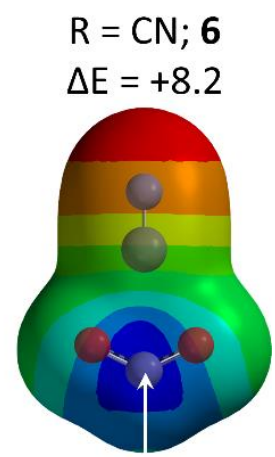

$+46$

Figure S2. Molecular Electrostatic Potential maps (MEPs) of structural isomers of $\mathrm{R}-\mathrm{NO}_{2}$ molecules derived from MP2/6-311+G** energy optimized geometries. The energetic values are in $\mathrm{kcal} / \mathrm{mol}$ and $\Delta \mathrm{E}$ quantifies by what margin these isomers are less stable.

\footnotetext{
${ }^{1}$ AIMAll (Version 13.05.06), Todd A. Keith, TK Gristmill Software, Overland Park KS, USA, 2013.
} 


\subsection{Cartesian coordinates of optimized geometries}

$\begin{array}{cccc}\mathbf{1} & & & \\ \mathrm{N} & 0.000000 & 0.000000 & 0.311591 \\ \mathrm{O} & -1.103108 & 0.000000 & -0.225237 \\ \mathrm{O} & 1.103108 & 0.000000 & -0.225237 \\ \mathrm{H} & 0.000000 & 0.000000 & 1.349735 \\ & & & \\ \mathbf{2} & & & \\ \mathrm{N} & 0.000000 & 0.000000 & 0.215671 \\ \mathrm{O} & 1.101106 & 0.000000 & 0.638775 \\ \mathrm{O} & -1.101106 & 0.000000 & 0.638775 \\ \mathrm{~F} & 0.000000 & 0.000000 & -1.320781\end{array}$

\section{Isomer of 2}

$\begin{array}{lrrl}\mathrm{N} & 0.942895 & 0.070015 & -0.000349 \\ \mathrm{O} & 0.364852 & 1.120568 & -0.000223 \\ \mathrm{O} & 0.528195 & -1.055088 & -0.000041 \\ \mathrm{~F} & -1.540346 & -0.116255 & 0.000152\end{array}$

3

$\begin{array}{llll}N & 0.000000 & 0.000000 & 0.596337\end{array}$

$\begin{array}{llll}0 & 1.096445 & 0.000000 & 1.071458\end{array}$

$\begin{array}{llll}0 & -1.096445 & 0.000000 & 1.071458\end{array}$

Cl $\quad 0.000000 \quad 0.000000 \quad-1.313409$

\section{Isomer of 3}

$\begin{array}{rrrr}\mathrm{N} & 1.399949 & -0.005056 & 0.001275 \\ \mathrm{O} & 0.947860 & 1.092225 & 0.000702 \\ \mathrm{O} & 0.938627 & -1.098485 & 0.000858 \\ \mathrm{Cl} & -1.463539 & 0.006991 & -0.002145\end{array}$

$\begin{array}{lrrr}4 & & & \\ \mathrm{~N} & 0.000000 & 0.000000 & 1.069689 \\ \mathrm{O} & 1.097775 & 0.000000 & 1.541105 \\ \mathrm{O} & -1.097775 & 0.000000 & 1.541105 \\ \mathrm{Br} & 0.000000 & 0.000000 & -1.034599\end{array}$

\section{Isomer of 4}
N $\quad 0.000000$
$0.000000 \quad 1.878510$
$\begin{array}{llll}0 & 1.095909 & 0.000000 & 1.434367\end{array}$
$\begin{array}{llll}0 & -1.095909 & 0.000000 & 1.434367\end{array}$
$\mathrm{Br} \quad 0.000000 \quad 0.000000 \quad-1.108279$
5
$\begin{array}{llll}\mathrm{N} & 0.000000 & 0.000000 & 1.380509\end{array}$
$\begin{array}{llll}\mathrm{O} & 1.096046 & 0.000000 & 1.869887\end{array}$ 


$$
\begin{array}{llll}
0 & -1.096046 & 0.000000 & 1.869887 \\
\text { I } & 0.000000 & 0.000000 & -0.929218
\end{array}
$$

\section{Isomer of 5}

$\begin{array}{cccc}\mathrm{N} & 0.000000 & 0.000000 & 2.163467 \\ \mathrm{O} & 1.093112 & 0.000000 & 1.719444 \\ \mathrm{O} & -1.093112 & 0.000000 & 1.719444 \\ \mathrm{I} & 0.000000 & 0.000000 & -1.006979\end{array}$

\section{6}

$\begin{array}{cccc}\mathrm{N} & 0.000000 & 0.000000 & 0.508928 \\ \mathrm{O} & 1.105770 & 0.000000 & 1.043793 \\ \mathrm{O} & -1.105770 & 0.000000 & 1.043793 \\ \mathrm{C} & 0.000000 & 0.000000 & -0.914891 \\ \mathrm{~N} & 0.000000 & 0.000000 & -2.092007\end{array}$

\section{Isomer of 6}

$\begin{array}{rrrr}\mathrm{N} & 1.359588 & 0.001876 & 0.002109 \\ \mathrm{O} & 0.838898 & 1.074296 & 0.001716 \\ \mathrm{O} & 0.841520 & -1.071821 & 0.002383 \\ \mathrm{C} & -1.135043 & -0.001443 & 0.002151 \\ \mathrm{~N} & -2.327455 & -0.004060 & 0.002709\end{array}$

\section{7}

$\begin{array}{llll}\text { C } & 0.000000 & 0.000000 & 0.245812\end{array}$

$\begin{array}{llll}\text { C } & 0.000000 & 0.000000 & -2.522837\end{array}$

$\begin{array}{llll}\text { C } & 1.224088 & 0.000000 & -0.423569\end{array}$

$\begin{array}{llll}\text { C } & -1.224088 & 0.000000 & -0.423569\end{array}$

$\begin{array}{llll}\text { C } & -1.212197 & 0.000000 & -1.821875\end{array}$

$\begin{array}{llll}\text { C } & 1.212197 & 0.000000 & -1.821875\end{array}$

$\begin{array}{llll}\text { H } & 2.149623 & 0.000000 & 0.140782\end{array}$

$\begin{array}{llll}H & -2.149623 & 0.000000 & 0.140782\end{array}$

$\begin{array}{llll}H & -2.154246 & 0.000000 & -2.362865\end{array}$

$\begin{array}{llll}H & 2.154246 & 0.000000 & -2.362865\end{array}$

$\begin{array}{llll}H & 0.000000 & 0.000000 & -3.609196\end{array}$

$\begin{array}{llll}\mathrm{N} & 0.000000 & 0.000000 & 1.727009\end{array}$

$\begin{array}{llll}\mathrm{O} & 1.092318 & 0.000000 & 2.295151\end{array}$

$\begin{array}{llll}0 & -1.092318 & 0.000000 & 2.295151\end{array}$

$\begin{array}{rrrr}\mathbf{8} & & & \\ \mathrm{N} & 1.520504 & 0.008986 & 0.000000 \\ \mathrm{O} & 1.390656 & 1.190146 & 0.000000 \\ \mathrm{O} & 2.431082 & -0.748963 & 0.000000 \\ \mathrm{O} & 0.184617 & -0.835882 & 0.000000 \\ \mathrm{C} & -0.966508 & -0.096497 & 0.000000 \\ \mathrm{~F} & -1.073639 & 0.684264 & 1.079519 \\ \mathrm{~F} & -1.073639 & 0.684264 & -1.079519\end{array}$




$\begin{array}{cccc}\text { F } & -1.968708 & -0.966972 & 0.000000 \\ \mathbf{9} & & & \\ \mathrm{N} & 0.611051 & -0.050505 & 0.000000 \\ \mathrm{O} & 1.631770 & 0.593046 & 0.000000 \\ \mathrm{O} & 0.443778 & -1.252336 & 0.000000 \\ \mathrm{O} & -0.544175 & 0.763464 & 0.000000 \\ \mathrm{C} & -1.758999 & 0.002167 & 0.000000 \\ \mathrm{H} & -1.833366 & -0.617763 & -0.894695 \\ \mathrm{H} & -2.531706 & 0.770187 & 0.000000 \\ \mathrm{H} & -1.833366 & -0.617763 & 0.894695\end{array}$

10

$\begin{array}{llll}\mathrm{N} & 0.955222 & -0.023521 & 0.000000\end{array}$

$\begin{array}{llll}\mathrm{O} & 1.486370 & -0.001022 & -1.102716\end{array}$

$\begin{array}{llll}0 & 1.486370 & -0.001022 & 1.102716\end{array}$

$\begin{array}{llll}\text { C } & -0.581856 & -0.007151 & 0.000000\end{array}$

$\begin{array}{llll}\text { F } & -0.961565 & 1.259848 & 0.000000\end{array}$

F $\quad-1.018213 \quad-0.615815 \quad-1.079448$

$\begin{array}{llll}F & -1.018213 & -0.615815 & 1.079448\end{array}$

11

$\begin{array}{llll}\mathrm{N} & 0.168427 & -0.014208 & 0.000000\end{array}$

$\begin{array}{llll}0 & 0.730192 & 0.003730 & -1.094678\end{array}$

$\begin{array}{llll}0 & 0.730192 & 0.003730 & 1.094678\end{array}$

$\begin{array}{llll}\text { C } & -1.324493 & -0.006649 & 0.000000\end{array}$

$\begin{array}{llll}\mathrm{H} & -1.660609 & -0.506976 & -0.905069\end{array}$

$\begin{array}{llll}H & -1.637070 & 1.037863 & 0.000000\end{array}$

$\begin{array}{llll}H & -1.660609 & -0.506976 & 0.905069\end{array}$

12

$\begin{array}{lrrr}\mathrm{N} & 0.925297 & 0.000000 & 0.000000 \\ \mathrm{O} & 1.383647 & 1.109424 & 0.000000 \\ \mathrm{O} & 1.383647 & -1.109424 & 0.000000 \\ \mathrm{~N} & -0.925297 & 0.000000 & 0.000000 \\ \mathrm{O} & -1.383647 & 1.109424 & 0.000000 \\ \mathrm{O} & -1.383647 & -1.109424 & 0.000000\end{array}$

$\left[\mathbf{2}+\mathbf{F}^{-}\right]^{-}$

$\begin{array}{llll}N & -0.032259 & 0.363818 & 0.000000\end{array}$

$\begin{array}{llll}0 & -0.040439 & 0.834885 & -1.096222\end{array}$

$\begin{array}{llll}0 & -0.040439 & 0.834885 & 1.096222\end{array}$

F $\quad-1.316953 \quad-0.872428 \quad 0.000000$

F $\quad 1.291902 \quad-0.829571 \quad 0.000000$

$\left[2+\mathrm{Cl}^{-}\right]^{-}$

$\begin{array}{llll}\text { N } & -1.049420 & 0.091636 & 0.000000\end{array}$ 


$\begin{array}{lrrr}\mathrm{O} & -0.784617 & 0.354047 & -1.105493 \\ \mathrm{O} & -0.784617 & 0.354047 & 1.105493 \\ \mathrm{~F} & -2.241351 & -1.147465 & 0.000000 \\ \mathrm{Cl} & 2.120555 & 0.566663 & 0.000000 \\ & & & \\ {\left[\mathbf{2}+\mathrm{NC}^{-}\right.} & & & \\ \mathrm{N} & -0.931069 & 0.101741 & -0.012771 \\ \mathrm{O} & -0.649143 & 0.353606 & -1.117051 \\ \mathrm{O} & -0.676469 & 0.375395 & 1.092690 \\ \mathrm{~F} & -2.107644 & -1.137553 & -0.014546 \\ \mathrm{~N} & 3.227633 & 0.335281 & 0.032420 \\ \mathrm{C} & 2.046631 & 0.167840 & 0.022656 \\ & & & \\ {[\mathbf{2}+\mathrm{NCCH}]} & & \\ \mathrm{N} & -1.092219 & -0.072646 & -0.005742 \\ \mathrm{O} & -1.318316 & 0.242848 & -1.113774 \\ \mathrm{O} & -1.349097 & 0.251716 & 1.092966 \\ \mathrm{~F} & -0.138125 & -1.354013 & 0.012777 \\ \mathrm{~N} & 1.233851 & 1.711635 & 0.005117 \\ \mathrm{C} & 2.016812 & 0.836159 & 0.004069 \\ \mathrm{C} & 2.973892 & -0.268604 & 0.002531 \\ \mathrm{H} & 2.424402 & -1.210921 & -0.022659 \\ \mathrm{H} & 3.587873 & -0.228158 & 0.904106 \\ \mathrm{H} & 3.620002 & -0.198061 & -0.874471\end{array}$

\section{[2+ $\left.\mathrm{O}\left(\mathrm{CH}_{3}\right)_{2}\right]$}

$\begin{array}{lrrr}\mathrm{N} & -1.239896 & 0.088971 & 0.000000 \\ \mathrm{O} & -1.346768 & 0.486053 & -1.101558 \\ \mathrm{O} & -1.346768 & 0.486053 & 1.101558 \\ \mathrm{~F} & -0.879678 & -1.437957 & 0.000000 \\ \mathrm{O} & 1.369121 & 0.503752 & 0.000000 \\ \mathrm{C} & 1.897289 & -0.106541 & 1.163431 \\ \mathrm{H} & 1.646298 & -1.174725 & 1.190040 \\ \mathrm{H} & 1.448904 & 0.391532 & 2.024151 \\ \mathrm{H} & 2.988378 & 0.010881 & 1.206428 \\ \mathrm{C} & 1.897289 & -0.106541 & -1.163431 \\ \mathrm{H} & 1.646298 & -1.174725 & -1.190040 \\ \mathrm{H} & 2.988378 & 0.010881 & -1.206428 \\ \mathrm{H} & 1.448904 & 0.391532 & -2.024151\end{array}$

\begin{tabular}{lrrr}
\multicolumn{4}{l}{$\left[6+\mathbf{F}^{-}\right]^{-}$} \\
N & -0.019580 & 0.718134 & -0.001019 \\
$\mathrm{O}$ & -0.134730 & 1.288621 & 1.090290 \\
$\mathrm{O}$ & -0.137450 & 1.285069 & -1.093889 \\
$\mathrm{C}$ & 0.426549 & -0.734591 & 0.000776 \\
$\mathrm{~N}$ & 1.528293 & -1.208325 & 0.000375 \\
$\mathrm{~F}$ & -0.926040 & -1.359866 & 0.003098
\end{tabular}




\begin{tabular}{llll}
\multicolumn{4}{l}{$\left[6+\mathrm{Cl}^{-}\right]^{-}$} \\
$\mathrm{N}$ & -0.932977 & -0.634131 & 0.000000 \\
$\mathrm{O}$ & -1.071395 & -1.175209 & -1.096304 \\
$\mathrm{O}$ & -1.071395 & -1.175209 & 1.096304 \\
$\mathrm{C}$ & -0.673591 & 0.790544 & 0.000000 \\
$\mathrm{~N}$ & -1.291750 & 1.813134 & 0.000000 \\
$\mathrm{Cl}$ & 1.618902 & 0.542191 & 0.000000
\end{tabular}

\begin{tabular}{llll}
\multicolumn{4}{l}{$\left.\mathbf{l}+\mathrm{NC}^{-}\right]^{-}$} \\
$\mathrm{N}$ & -0.761552 & -0.997938 & 0.058772 \\
$\mathrm{O}$ & -1.553762 & -1.280534 & -0.878269 \\
$\mathrm{O}$ & -0.750072 & -1.752600 & 1.066874 \\
$\mathrm{C}$ & 0.239297 & 1.422873 & -0.205383 \\
$\mathrm{~N}$ & -0.537759 & 2.274690 & -0.467511 \\
$\mathrm{C}$ & 1.327609 & 0.617916 & 0.090115 \\
$\mathrm{~N}$ & 2.369747 & 0.113719 & 0.329714
\end{tabular}

[6 + $\mathrm{NCCH}_{3}$ ]

$\begin{array}{llll}\mathrm{N} & -1.568510 & -0.638812 & -0.000478\end{array}$

$\begin{array}{llll}\mathrm{O} & -1.569159 & -1.176734 & -1.103849\end{array}$

$\begin{array}{llll}0 & -1.580113 & -1.173322 & 1.104507\end{array}$

$\begin{array}{llll}\text { C } & -1.569624 & 0.782090 & -0.002937\end{array}$

$\begin{array}{llll}\text { N } & -1.654423 & 1.956424 & -0.005521\end{array}$

$\begin{array}{llll}\mathrm{N} & 1.214506 & 0.016479 & 0.010431\end{array}$

$\begin{array}{llll}\text { C } & 2.380533 & 0.142575 & 0.007432\end{array}$

$\begin{array}{llll}\text { C } & 3.833527 & 0.301216 & 0.003655\end{array}$

$\begin{array}{llll}\text { H } & 4.252266 & -0.149853 & -0.897726\end{array}$

$\begin{array}{llll}H & 4.261040 & -0.187579 & 0.880927\end{array}$

$\begin{array}{llll}\text { H } & 4.088681 & 1.362261 & 0.024891\end{array}$

\begin{tabular}{llll}
\multicolumn{4}{l}{$\left.\mathbf{6}+\mathrm{O}\left(\mathrm{CH}_{3}\right)_{2}\right]$} \\
$\mathrm{N}$ & -1.312456 & -0.401349 & -0.002199 \\
$\mathrm{O}$ & -1.491876 & -0.899414 & -1.109646 \\
$\mathrm{O}$ & -1.491556 & -0.912154 & 1.099469 \\
$\mathrm{C}$ & -0.849316 & 0.942241 & 0.005448 \\
$\mathrm{~N}$ & -0.556126 & 2.083772 & 0.011967 \\
$\mathrm{O}$ & 1.359401 & -0.516192 & -0.002753 \\
$\mathrm{C}$ & 2.041835 & -0.099465 & 1.167192 \\
$\mathrm{H}$ & 2.114317 & 0.995987 & 1.208749 \\
$\mathrm{H}$ & 3.051719 & -0.527730 & 1.203270 \\
$\mathrm{H}$ & 1.466632 & -0.459886 & 2.021313 \\
$\mathrm{C}$ & 2.041301 & -0.087063 & -1.168525 \\
$\mathrm{H}$ & 2.113812 & 1.008766 & -1.198462 \\
$\mathrm{H}$ & 1.465685 & -0.438357 & -2.026165 \\
$\mathrm{H}$ & 3.051152 & -0.514953 & -1.209635
\end{tabular}




\begin{tabular}{|c|c|c|c|}
\hline \multicolumn{4}{|c|}{$\left[8+F^{-}\right]^{-}$} \\
\hline $\mathrm{N}$ & -1.771168 & -0.373085 & 0.302396 \\
\hline $\mathrm{O}$ & -1.590163 & -1.279187 & -0.411319 \\
\hline 0 & -1.252719 & 0.304875 & 1.097668 \\
\hline $\mathrm{O}$ & 0.550922 & 0.451690 & -1.096696 \\
\hline C & 1.472137 & 0.200938 & -0.334474 \\
\hline $\mathrm{F}$ & 1.727058 & 1.143875 & 0.702585 \\
\hline $\mathrm{F}$ & 1.344776 & -1.011230 & 0.434483 \\
\hline $\mathrm{F}$ & 2.760649 & 0.037451 & -0.894182 \\
\hline 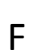 & -3.378524 & 0.128503 & 0.130262 \\
\hline
\end{tabular}

\begin{tabular}{lrrr}
\multicolumn{4}{l}{$\left[8+\mathrm{Cl}^{-}\right]^{-}$} \\
$\mathrm{N}$ & -0.697424 & 0.615865 & 0.034770 \\
$\mathrm{O}$ & -1.409653 & -0.189935 & 0.550771 \\
$\mathrm{O}$ & -0.880974 & 1.757778 & -0.256323 \\
$\mathrm{O}$ & 0.978416 & -0.259065 & -1.163764 \\
$\mathrm{C}$ & 1.834768 & -0.468191 & -0.276435 \\
$\mathrm{~F}$ & 2.174828 & 0.643195 & 0.517848 \\
$\mathrm{~F}$ & 1.491102 & -1.423992 & 0.691511 \\
$\mathrm{~F}$ & 3.070471 & -0.914190 & -0.739254 \\
$\mathrm{Cl}$ & -3.378277 & 1.561759 & 0.522217
\end{tabular}

\begin{tabular}{lrrr}
\multicolumn{4}{l}{$\mathbf{8}+\mathbf{N C}^{-}$} \\
$\mathrm{N}$ & 0.888277 & 0.055003 & 0.002622 \\
$\mathrm{O}$ & 1.368836 & 0.774790 & -0.811763 \\
$\mathrm{O}$ & 1.403420 & -0.645323 & 0.812901 \\
$\mathrm{O}$ & -1.094965 & -0.813948 & -0.720855 \\
$\mathrm{C}$ & -1.892899 & -0.141749 & -0.044667 \\
$\mathrm{~F}$ & -1.754687 & -0.250215 & 1.356775 \\
$\mathrm{~F}$ & -1.826991 & 1.257923 & -0.228792 \\
$\mathrm{~F}$ & -3.246965 & -0.415719 & -0.243762 \\
$\mathrm{C}$ & 3.457971 & 0.000248 & -0.106674 \\
$\mathrm{~N}$ & 4.644763 & 0.061178 & -0.094965 \\
& & & \\
{$[\mathbf{8}+\mathbf{N C C H}]$} & & \\
$\mathrm{N}$ & 0.234083 & 1.543731 & -0.168227 \\
$\mathrm{O}$ & 0.506057 & 1.624101 & 0.972921 \\
$\mathrm{O}$ & -0.009406 & 2.226849 & -1.090651 \\
$\mathrm{O}$ & 0.122025 & -0.079943 & -0.755295 \\
$\mathrm{C}$ & 0.612403 & -0.989468 & 0.098008 \\
$\mathrm{~F}$ & 1.894438 & -0.758016 & 0.437134 \\
$\mathrm{~F}$ & -0.082567 & -1.059605 & 1.254020 \\
$\mathrm{~F}$ & 0.544439 & -2.191118 & -0.486549 \\
$\mathrm{~N}$ & -2.563421 & 1.530349 & 0.502389 \\
$\mathrm{C}$ & -2.803055 & 0.423862 & 0.188629 \\
$\mathrm{C}$ & -3.085810 & -0.954057 & -0.208043 \\
$\mathrm{H}$ & -4.145408 & -1.061832 & -0.448073
\end{tabular}




$$
\begin{array}{llll}
H & -2.828039 & -1.631340 & 0.608215 \\
H & -2.484859 & -1.206360 & -1.083764
\end{array}
$$

$$
\left[8+\mathrm{O}\left(\mathrm{CH}_{3}\right)_{2}\right]
$$

$\begin{array}{rrrr}\mathrm{N} & 0.210461 & 1.460840 & 0.230494 \\ \mathrm{O} & 0.324111 & 1.186438 & 1.372222 \\ \mathrm{O} & -0.192057 & 2.349545 & -0.426745 \\ \mathrm{O} & 0.800835 & 0.321413 & -0.829778 \\ \mathrm{C} & 1.300414 & -0.781166 & -0.236888 \\ \mathrm{~F} & 2.345473 & -0.517625 & 0.566686 \\ \mathrm{~F} & 0.393244 & -1.436771 & 0.510403 \\ \mathrm{~F} & 1.712807 & -1.605032 & -1.202253 \\ \mathrm{O} & -2.152473 & 0.318659 & 0.108974 \\ \mathrm{C} & -2.675117 & -0.484808 & 1.150318 \\ \mathrm{H} & -3.768206 & -0.560978 & 1.074761 \\ \mathrm{H} & -2.413417 & -0.004016 & 2.094152 \\ \mathrm{H} & -2.241673 & -1.492885 & 1.124199 \\ \mathrm{C} & -2.419933 & -0.254360 & -1.158392 \\ \mathrm{H} & -3.501500 & -0.326299 & -1.334916 \\ \mathrm{H} & -1.975583 & -1.255261 & -1.236628 \\ \mathrm{H} & -1.972620 & 0.396886 & -1.909919\end{array}$

\begin{tabular}{|c|c|c|c|}
\hline \multicolumn{4}{|c|}{$\left[10+\mathrm{NC}^{-}\right]^{-}$} \\
\hline $\mathrm{N}$ & 0.277582 & 1.007469 & 0.031151 \\
\hline & 0.157257 & 1.501370 & 1.142890 \\
\hline & 0.185749 & 1.558572 & -1.056106 \\
\hline & 0.810286 & -0.423185 & 0.000779 \\
\hline
\end{tabular}

$$
\begin{aligned}
& {\left[10+\mathrm{F}^{-}\right]^{-}} \\
& \text {N } \quad-0.314653 \quad 0.845325 \quad-0.001593 \\
& \begin{array}{llll}
0 & -0.479979 & 1.368389 & 1.095208
\end{array} \\
& \begin{array}{llll}
0 & -0.484930 & 1.362065 & -1.100642
\end{array} \\
& \begin{array}{llll}
\text { C } & 0.552714 & -0.394019 & -0.000034
\end{array} \\
& \begin{array}{llll}
\text { F } & 1.830327 & 0.105964 & -0.004179
\end{array} \\
& \begin{array}{llll}
\text { F } & 0.433907 & -1.107044 & 1.090082
\end{array} \\
& \text { F } \quad \begin{array}{llll}
0.429211 & -1.113087 & -1.085633
\end{array} \\
& \begin{array}{llll}
\text { F } & -1.882883 & -0.693947 & 0.006149
\end{array}
\end{aligned}
$$

\begin{tabular}{lrrr}
\multicolumn{4}{l}{$\left[10+\mathrm{Cl}^{-}\right]^{-}$} \\
$\mathrm{N}$ & 0.458194 & 0.969722 & 0.000000 \\
$\mathrm{O}$ & 0.393445 & 1.499926 & 1.100043 \\
$\mathrm{O}$ & 0.393445 & 1.499926 & -1.100043 \\
$\mathrm{C}$ & 0.900738 & -0.491143 & 0.000000 \\
$\mathrm{~F}$ & 2.256061 & -0.424566 & 0.000000 \\
$\mathrm{~F}$ & 0.522257 & -1.107920 & 1.084951 \\
$\mathrm{~F}$ & 0.522257 & -1.107920 & -1.084951 \\
$\mathrm{Cl}$ & -2.315422 & -0.249446 & 0.000000
\end{tabular}




$\begin{array}{llll}\mathrm{F} & 2.157374 & -0.277045 & 0.021608 \\ \mathrm{~F} & 0.453618 & -1.089897 & 1.064185 \\ \mathrm{~F} & 0.480896 & -1.033416 & -1.104515 \\ \mathrm{C} & -2.316134 & -0.374092 & -0.038498 \\ \mathrm{~N} & -3.463677 & -0.701377 & -0.064529 \\ & & & \\ \left.\mathrm{C} \mathbf{1 0}+\mathbf{N C C H}_{3}\right] & & \\ \mathrm{N} & 0.114662 & 0.713382 & 0.305783 \\ \mathrm{O} & -0.009587 & 0.918653 & 1.505312 \\ \mathrm{O} & -0.503935 & 1.227135 & -0.618117 \\ \mathrm{C} & 1.228814 & -0.259533 & -0.105049 \\ \mathrm{~F} & 2.313040 & 0.476457 & -0.323449 \\ \mathrm{~F} & 1.444889 & -1.109584 & 0.865957 \\ \mathrm{~F} & 0.875025 & -0.878128 & -1.206075 \\ \mathrm{~N} & -1.747388 & -1.608406 & 0.437427 \\ \mathrm{C} & -2.644029 & -0.898493 & 0.170487 \\ \mathrm{C} & -3.736577 & 0.014079 & -0.160945 \\ \mathrm{H} & -3.319961 & 0.944033 & -0.553614 \\ \mathrm{H} & -4.320606 & 0.232328 & 0.735106 \\ \mathrm{H} & -4.385857 & -0.439431 & -0.912086\end{array}$

\begin{tabular}{lrrr}
\multicolumn{4}{l}{$\left.\mathbf{1 0}+\mathbf{O}\left(\mathrm{CH}_{3}\right)_{2}\right]$} \\
$\mathrm{N}$ & -0.622799 & 0.799305 & -0.421431 \\
$\mathrm{O}$ & -0.465552 & 0.743705 & -1.633193 \\
$\mathrm{O}$ & -0.461387 & 1.757614 & 0.321097 \\
$\mathrm{C}$ & -1.195605 & -0.461658 & 0.233713 \\
$\mathrm{~F}$ & -2.519368 & -0.343207 & 0.174973 \\
$\mathrm{~F}$ & -0.812914 & -1.522464 & -0.435930 \\
$\mathrm{~F}$ & -0.808411 & -0.525914 & 1.484685 \\
$\mathrm{O}$ & 1.736777 & -0.311721 & 0.171147 \\
$\mathrm{C}$ & 2.479393 & -0.581729 & -1.003413 \\
$\mathrm{H}$ & 2.731396 & 0.347226 & -1.532513 \\
$\mathrm{H}$ & 1.853621 & -1.202241 & -1.645713 \\
$\mathrm{H}$ & 3.405922 & -1.120654 & -0.764737 \\
$\mathrm{C}$ & 2.467713 & 0.506325 & 1.066071 \\
$\mathrm{H}$ & 2.717921 & 1.470504 & 0.603356 \\
$\mathrm{H}$ & 3.394492 & 0.008881 & 1.381420 \\
$\mathrm{H}$ & 1.834023 & 0.679608 & 1.936487
\end{tabular}

\begin{tabular}{|c|c|c|c|}
\hline \multicolumn{4}{|c|}{$\left[12+\mathrm{F}^{-}\right]^{-}$} \\
\hline$N$ & -0.727286 & 0.570528 & 0.001139 \\
\hline 0 & -1.152882 & 0.799928 & 1.103112 \\
\hline $\mathrm{O}$ & -1.150730 & 0.807248 & -1.100115 \\
\hline$N$ & 0.927600 & 0.009710 & 0.000895 \\
\hline $\mathrm{O}$ & 1.404988 & -0.070232 & 1.102708 \\
\hline $\mathrm{O}$ & 1.407146 & -0.062911 & -1.100488 \\
\hline $\mathrm{F}$ & -0.573601 & -1.672593 & -0.006170 \\
\hline
\end{tabular}




\begin{tabular}{llll}
\multicolumn{4}{l}{$\left[\mathbf{1 2}+\mathrm{Cl}^{-}\right]^{-}$} \\
$\mathrm{N}$ & 0.898329 & 0.000000 & -0.811843 \\
$\mathrm{O}$ & 1.364058 & -1.104741 & -0.868549 \\
$\mathrm{O}$ & 1.364058 & 1.104741 & -0.868549 \\
$\mathrm{~N}$ & -0.898329 & 0.000000 & -0.811843 \\
$\mathrm{O}$ & -1.364058 & -1.104741 & -0.868549 \\
$\mathrm{O}$ & -1.364058 & 1.104741 & -0.868549 \\
$\mathrm{Cl}$ & 0.000000 & 0.000000 & 2.069159
\end{tabular}

\begin{tabular}{lrrr}
\multicolumn{4}{l}{$\left.\begin{array}{l}\text { [12 } \\
\text { N N }\end{array}\right]^{-}$} \\
0.688830 & 0.920641 & -0.015221 \\
$\mathrm{O}$ & 0.745851 & 1.391319 & 1.086910 \\
$\mathrm{O}$ & 0.687293 & 1.382473 & -1.122630 \\
$\mathrm{~N}$ & 0.744126 & -0.866008 & -0.009792 \\
$\mathrm{O}$ & 0.829826 & -1.325677 & 1.095203 \\
$\mathrm{O}$ & 0.771146 & -1.333865 & -1.114408 \\
$\mathrm{C}$ & -2.047228 & -0.060806 & 0.056334 \\
$\mathrm{~N}$ & -3.239122 & -0.126211 & 0.036973
\end{tabular}

\begin{tabular}{lrrr}
\multicolumn{4}{l}{$\left.\mathbf{1 2}+\mathrm{NCCH}_{3}\right]$} \\
$\mathrm{N}$ & -1.325559 & 0.905303 & 0.002504 \\
$\mathrm{O}$ & -1.326482 & 1.366490 & -1.105130 \\
$\mathrm{O}$ & -1.314791 & 1.366725 & 1.110016 \\
$\mathrm{~N}$ & -1.334356 & -0.913447 & 0.002651 \\
$\mathrm{O}$ & -1.339757 & -1.374514 & -1.104996 \\
$\mathrm{O}$ & -1.328038 & -1.374510 & 1.110319 \\
$\mathrm{~N}$ & 1.416385 & -0.016678 & -0.010122 \\
$\mathrm{C}$ & 2.589188 & 0.000826 & -0.005533 \\
$\mathrm{C}$ & 4.050736 & 0.019722 & -0.001042 \\
$\mathrm{H}$ & 4.407148 & 0.926031 & 0.491828 \\
$\mathrm{H}$ & 4.423964 & -0.000800 & -1.026515 \\
$\mathrm{H}$ & 4.430036 & -0.851784 & 0.535617
\end{tabular}

\begin{tabular}{|c|c|c|c|}
\hline \multicolumn{4}{|c|}{$\left[12+\mathrm{O}\left(\mathrm{CH}_{3}\right)_{2}\right]$} \\
\hline$N$ & 0.000000 & -0.907153 & 1.033825 \\
\hline 0 & 1.107419 & -1.368514 & 1.028231 \\
\hline $\mathrm{O}$ & -1.107419 & -1.368514 & 1.028231 \\
\hline $\mathrm{N}$ & 0.000000 & 0.907153 & 1.033825 \\
\hline 0 & 1.107419 & 1.368514 & 1.028231 \\
\hline 0 & -1.107419 & 1.368514 & 1.028231 \\
\hline $\mathrm{O}$ & 0.000000 & 0.000000 & -1.447355 \\
\hline C & 1.172059 & 0.000000 & -2.240611 \\
\hline $\mathrm{H}$ & 1.215190 & 0.894259 & -2.876561 \\
\hline $\mathrm{H}$ & 1.215190 & -0.894259 & -2.876561 \\
\hline $\mathrm{H}$ & 2.022613 & 0.000000 & -1.557374 \\
\hline C & -1.172059 & 0.000000 & -2.240611 \\
\hline
\end{tabular}


$\begin{array}{llll}H & -1.215190 & 0.894259 & -2.876561\end{array}$

$\begin{array}{llll}\mathrm{H} & -2.022613 & 0.000000 & -1.557374\end{array}$

$\begin{array}{llll}H & -1.215190 & -0.894259 & -2.876561\end{array}$ 


\section{CSD Analysis}

\subsection{Description of queries used to retrieve data from the CSD}

The CSD (version 5.37 (Nov. 2015 including two updates) was inspected using ConQuest (version 1.18). All searches were limited to high quality structures $(R \leq 0.1)$ and powder structures and structures containing errors were omitted.

Two of the $\mathrm{N}-\mathrm{O}$ bonds were set as 'any type', while the $\mathrm{N}-\mathrm{O} 2 \mathrm{C}$ bond was set as a single bond. All covalent bond distances and selected triatomic angles were collected to reconstruct the average models used for the inset nitrate ester models in Figure 3, Figure S4 and Figure S5. The interatomic distance between the interacting atom $(\mathrm{F}, \mathrm{Cl}, \mathrm{Br}, \mathrm{I}, \mathrm{At}, \mathrm{O}, \mathrm{S}$, $\mathrm{Se}, \mathrm{Te}, \mathrm{N}, \mathrm{P}$ or As in EIR ('electron rich') or $\mathrm{O}$ in $\mathrm{O}=\mathrm{X}$ and the nitro's $\mathrm{N}$-atom (e, highlighted in red in Figure S3) was set as $\leq 5 \AA$ so that the data was confined within a $10 \AA$ diameter sphere centred on $\mathrm{N}$.

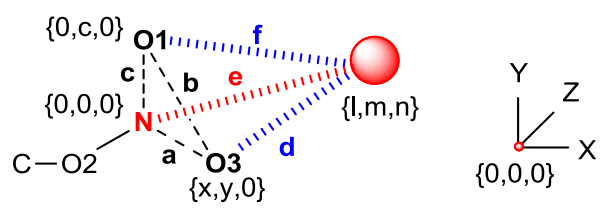

Figure S3. Illustration of the query used to extract (geometric) data from the PDB and CSD.

\subsection{Deriving geometric parameters $(X Y Z, r)$}

The interatomic distances between the interacting atom and 01 and 03 , as well as the 01 $\mathrm{O} 3$ distance were also collected. The triangle formed by $\mathrm{O} 1-\mathrm{N}-\mathrm{O} 3$ was chosen as the base, and the interacting atom as the tip of a tetrahedron (see Figure S3) so that Cartesian Coordinates $\{X, Y, Z\}$ of all the atoms could be derived as follows: the $\mathrm{N}$-atom was taken as the centre $\{0,0,0\}, 01$ as $\{0, c, 0\}, 03$ as $\{x, y, 0\}$, and the interacting atom at $\{\mathbf{I}, \mathbf{m}, \mathbf{n}\}$. Distances a-f were measured, from which $\mathbf{y}, \mathbf{x}, \mathbf{m}, \mathbf{I}$ and $\mathbf{n}$ can be derived using equations (S1) - (S5) respectively.

$$
\begin{array}{ll}
y=\frac{a^{2}+c^{2}-b^{2}}{2 c} & \\
x=\sqrt{a^{2}-y^{2}} & \quad(=\text { X-value) } \\
m=\frac{c^{2}+e^{2}-d^{2}}{2 c} & \text { (= Y-value) } \\
l=\frac{a^{2}+e^{2}-f^{2}-2 m y}{2 x} & \text { (= Z-value) }
\end{array}
$$

Thus, the distance between the interacting atom and the plane defined by $\mathrm{O} 1-\mathrm{N}-\mathrm{O} 3$ is $\mathbf{n}$, i.e. the Z-value. With this and the $\mathrm{N} \cdots$ interacting atom distance (e) the parallel displacement parameter $(\boldsymbol{r})$ could be derived according to equation (S6):

$$
r=\sqrt{e^{2}-n^{2}}
$$


With this procedure the sign of $\mathbf{n}$ (i.e. the Z-axis) is always positive, meaning that data in one half of the sphere were reflected to the other half of the sphere to obtain the data within a $5 \AA$ high and $10 \AA$ wide hemisphere.

To obtain all $\{X, Y, Z\}$ coordinates of the average models, it was assumed that $\mathrm{O} 2$ and $\mathrm{C}$ were coplanar with $01-\mathrm{N}-03$. The averages of relevant distances and angles were then used together with the rules of sine and cosine to obtain the $\{X, Y, Z\}$ coordinates. The relative standard deviations of the parameters used were typically below $5 \%$.

\subsection{Additional CSD data}

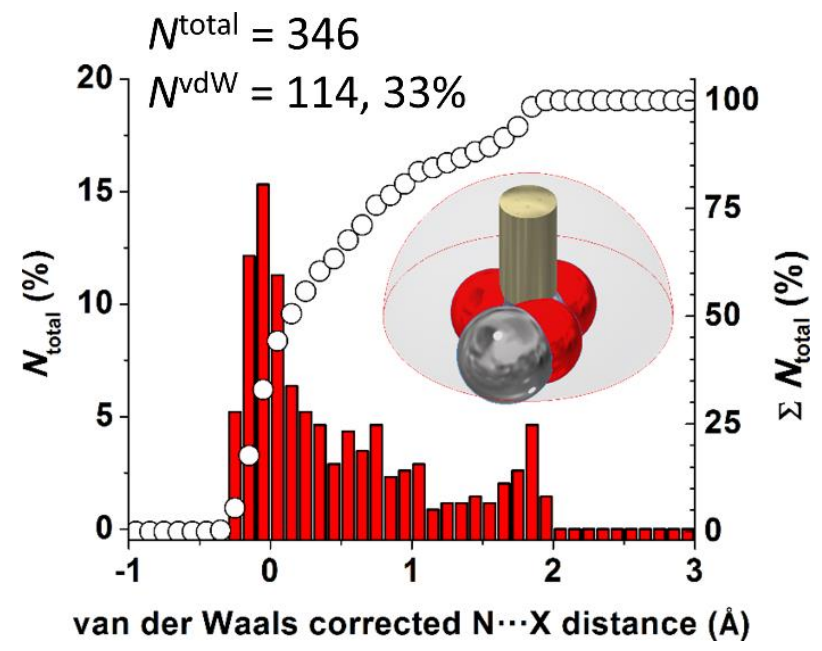

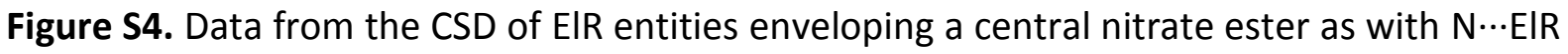
$\leq 5 \AA$. Within these data, 346 hits were characterized by a parallel displacement of $\leq 1 \AA$ from $\mathrm{N}$ (golden body in the inset figure) and these data were plotted as a function of the van der Waals corrected N...EIR distance). Of these data, 33\% (114) hits displayed van der Waals overlap.
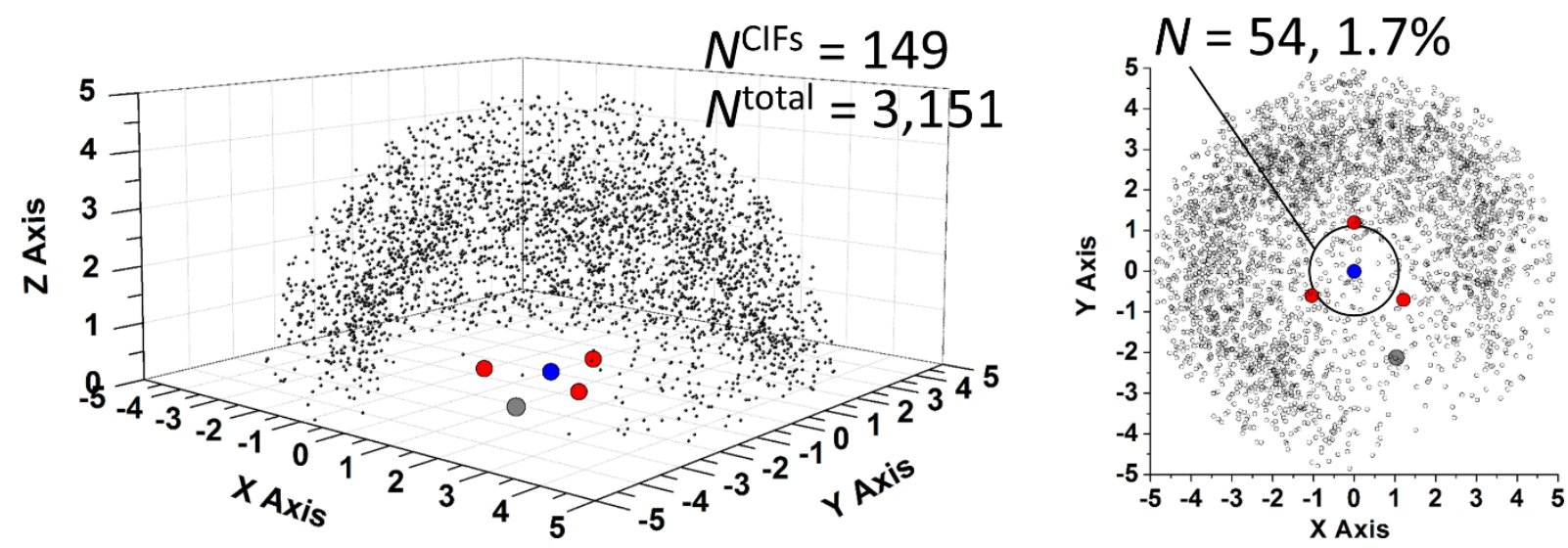

Figure S5. Perspective (left) and top (right) views of the distribution of $\mathrm{H}$-atoms (of $\mathrm{X}-\mathrm{H}$ ) enveloping a central nitrate ester as found within the CSD with $\mathrm{N} \cdots \mathrm{H} \leq 5 \AA$. These data span 3,515 hits found in 149 Crystallographic Information Files (CIFs). Of these data, only $1.7 \%$ (54 hits) are characterized by a parallel displacement of $\leq 1 \AA$ from $N$ (black circle in the righthand figure). 